BARI-TH/94-174

DSF-T-94/12

INFN-NA-IV-94/12

\title{
Form factor $A_{0}\left(q^{2}\right)$, nonleptonic $D(B) \rightarrow P V$ transitions and rare $B \rightarrow K^{*} \gamma$ decays
}

\author{
Pietro Colangelo ${ }^{1}$, Fulvia De Fazio ${ }^{1,2}$ and Pietro Santorelli ${ }^{3}$ \\ ${ }^{1}$ Istituto Nazionale di Fisica Nucleare, \\ Sezione di Bari, Italy \\ 2 Dipartimento di Fisica dell'Università di Bari, \\ via G.Amendola 173, 70126 Bari, Italy \\ 3 Dipartimento di Fisica dell'Università "Federico II" di Napoli \\ and Istituto Nazionale di Fisica Nucleare, Sezione di Napoli, \\ Mostra D'Oltremare, Pad 19-20, 80125 Napoli, Italy
}

(August 25, 1994)

\begin{abstract}
We use three-point function QCD sum rules to calculate the form factor $A_{0}\left(q^{2}\right)$ appearing in the matrix element of the flavour-changing axial current between the $D(B)$ state and a vector meson state. We describe the role of this form factor in nonleptonic $D(B) \rightarrow P V$ decays and analyze the light $S U(3)_{F}$ symmetry breaking effects. We also discuss a proposal to relate the branching ratio of $B \rightarrow K^{*} \gamma$ to the spectrum of the semileptonic $B \rightarrow \rho \ell \nu$ decay.
\end{abstract}

PACS:11.50.Li, 13.25

Typeset using REVTEX 


\section{INTRODUCTION}

The theoretical description of weak exclusive nonleptonic decays of heavy mesons is carried out, in the factorization approximation, in three steps. First, an effective hamiltonian is constructed taking into account the effects of hard gluon exchanges [1]. Then, the hadronic matrix elements are factorized into the product of current-particle matrix elements that can be either inferred from experiment or calculated theoretically (notice that we only consider two body decays) [2,3]. Finally, strong rescattering effects (mainly for $D$ meson decays) are included considering the coupling to intermediate resonances [4] or using the measured phase shifts [5].

The second step is dictated by our inability in reliably computing matrix elements of the effective hamiltonian between the external states involved in the decay. Let us con-

sider, for example, the transition $D^{+}(p) \rightarrow \bar{K}^{* 0}\left(p^{\prime}\right) \pi^{+}(q)$, which is governed by the effective hamiltonian:

$$
\mathcal{H}_{W}=-\frac{G_{F}}{\sqrt{2}} V_{u d}^{*} V_{c s}\left[\frac{c_{+}+c_{-}}{2} O_{1}+\frac{c_{+}-c_{-}}{2} O_{2}\right]
$$

where $G_{F}$ is the Fermi constant, $V_{h k}$ are CKM matrix elements and the local operators $O_{1}$ and $\mathrm{O}_{2}$ are given by:

$$
\begin{aligned}
& O_{1}=\bar{s} \gamma_{\mu}\left(1-\gamma_{5}\right) c \bar{u} \gamma^{\mu}\left(1-\gamma_{5}\right) d \\
& O_{2}=\bar{s} \gamma_{\mu}\left(1-\gamma_{5}\right) d \bar{u} \gamma^{\mu}\left(1-\gamma_{5}\right) c .
\end{aligned}
$$

The Wilson coefficients $c_{+}(\mu)$ and $c_{-}(\mu)$ account for the hard gluon effects in the renormalization of $\mathcal{H}_{W}$ from $m_{W}$ to the low energy scale $\mu$; they are known at the next-to-leading order approximation [6]. The amplitude $\mathcal{A}\left(D^{+} \rightarrow \bar{K}^{* 0} \pi^{+}\right)$is written, in the factorization approximation, as follows:

$$
\begin{aligned}
& \mathcal{A}\left(D^{+} \rightarrow \bar{K}^{* 0} \pi^{+}\right)_{\text {fact }}=-\frac{G_{F}}{\sqrt{2}} V_{u d}^{*} V_{c s}<\bar{K}^{* 0} \pi^{+}\left|\left[c_{1} O_{1}+c_{2} O_{2}\right]\right| D^{+}>_{\text {fact }} \\
& \equiv-\frac{G_{F}}{\sqrt{2}} V_{u d}^{*} V_{c s}\left\{\left(c_{1}+\frac{c_{2}}{N_{c}}\right)<\bar{K}^{* 0}\left|\bar{s} \gamma_{\mu}\left(1-\gamma_{5}\right) c\right| D^{+}><\pi^{+}\left|\bar{u} \gamma^{\mu}\left(1-\gamma_{5}\right) d\right| 0>\right. \\
& \left.+\left(c_{2}+\frac{c_{1}}{N_{c}}\right)<\pi^{+}\left|\bar{u} \gamma^{\mu}\left(1-\gamma_{5}\right) c\right| D^{+}><\bar{K}^{* 0}\left|\bar{s} \gamma_{\mu}\left(1-\gamma_{5}\right) d\right| 0>\right\}
\end{aligned}
$$

where $c_{1}=\left(c_{+}+c_{-}\right) / 2, c_{2}=\left(c_{+}-c_{-}\right) / 2$. To obtain (1.4) we have used the identities

$$
\begin{aligned}
& O_{1}=\frac{1}{N_{c}} O_{2}+\frac{1}{2}\left[\bar{s} \gamma_{\mu}\left(1-\gamma_{5}\right) \lambda^{a} d\right]\left[\bar{u} \gamma^{\mu}\left(1-\gamma_{5}\right) \lambda^{a} c\right] \\
& O_{2}=\frac{1}{N_{c}} O_{1}+\frac{1}{2}\left[\bar{s} \gamma_{\mu}\left(1-\gamma_{5}\right) \lambda^{a} c\right]\left[\bar{u} \gamma^{\mu}\left(1-\gamma_{5}\right) \lambda^{a} d\right]
\end{aligned}
$$

where $N_{c}$ is the number of colours and $\lambda^{a}$ are the Gell-Mann $S U(3)_{c}$ matrices. Therefore, the amplitude is expressed in terms of current-particle matrix elements 


$$
\begin{gathered}
<\bar{K}^{* 0}\left(p^{\prime}, \lambda\right)\left|\bar{s} \gamma_{\mu} d\right| 0>=f_{K^{*}} m_{K^{*}} \epsilon_{\mu}^{*}(\lambda) \\
<\pi^{+}(q)\left|\bar{u} \gamma_{\mu} \gamma_{5} d\right| 0>=-i f_{\pi} q_{\mu}
\end{gathered}
$$

$\left(\epsilon(\lambda)\right.$ is the $K^{*}$ polarization vector) and of the matrix elements governing the semileptonic transition $D \rightarrow K^{*}(\pi) \ell \nu$ :

$$
\begin{gathered}
<\bar{K}^{* 0}\left(p^{\prime}, \lambda\right)\left|\bar{s} \gamma_{\mu}\left(1-\gamma_{5}\right) c\right| D^{+}(p)>=\left\{\epsilon_{\mu \nu \rho \sigma} \epsilon^{* \nu} p^{\rho} p^{\prime \sigma} \frac{2 V\left(q^{2}\right)}{m_{D}+m_{K^{*}}}\right. \\
-i\left[\left(m_{D}+m_{K^{*}}\right) A_{1}\left(q^{2}\right) \epsilon_{\mu}^{*}-\frac{A_{2}\left(q^{2}\right)}{m_{D}+m_{K^{*}}}\left(\epsilon^{*} \cdot p\right)\left(p+p^{\prime}\right)_{\mu}\right. \\
\left.\left.-\left(\epsilon^{*} \cdot p\right) \frac{2 m_{K^{*}}}{q^{2}} q_{\mu}\left(A_{3}\left(q^{2}\right)-A_{0}\left(q^{2}\right)\right)\right]\right\} \\
<\pi^{+}(q)\left|\bar{u} \gamma_{\mu} c\right| D^{+}(p)>=(p+q)_{\mu} F_{1}\left(p^{\prime 2}\right)+\frac{m_{D}^{2}-m_{\pi}^{2}}{p^{2}}\left[F_{0}\left(p^{\prime 2}\right)-F_{1}\left(p^{\prime 2}\right)\right] p_{\mu}^{\prime}
\end{gathered}
$$

$\left(q=p-p^{\prime}\right)$. Notice that we have used the BSW [7] parameterization of the form factors; in this parameterization $A_{1}, A_{2}$ and $A_{3}$ are not independent, but they fulfil the relation:

$$
A_{3}\left(q^{2}\right)=\frac{m_{D}+m_{K^{*}}}{2 m_{K^{*}}} A_{1}\left(q^{2}\right)-\frac{m_{D}-m_{K^{*}}}{2 m_{K^{*}}} A_{2}\left(q^{2}\right)
$$

with

$$
A_{3}(0)=A_{0}(0)
$$

in order to avoid the unphysical singularity at $q^{2}=0$ in eq.(1.8); for the same reason $F_{0}(0)=F_{1}(0)$.

The amplitude of the process we are considering is therefore reduced to:

$$
\begin{aligned}
\mathcal{A}\left(D^{+} \rightarrow \bar{K}^{* 0} \pi^{+}\right)_{\text {fact }}=-\frac{G_{F}}{\sqrt{2}} V_{u d}^{*} V_{c s} 2 m_{K^{*}}\left(\epsilon^{*} \cdot p\right) & \left\{\left(c_{1}+\frac{c_{2}}{N_{c}}\right) f_{\pi} A_{0}\left(m_{\pi}^{2}\right)\right. \\
& \left.+\left(c_{2}+\frac{c_{1}}{N_{c}}\right) f_{K^{*}} F_{1}\left(m_{K^{*}}^{2}\right)\right\}
\end{aligned}
$$

The leptonic constants $f_{\pi}$ and $f_{K^{*}}$ can be derived from experiment. As for the Wilson coefficients, it has been observed [3,8] that consistently neglecting all $1 / N_{c}$ terms in the factorized amplitudes like eq.(1.12), and using the form factors e.g. from the BSW model [7], the overall agreement with the available experimental data seems to improve. This is an appealing feature of this approach, since factorization becomes exact in the multicolor chromodynamics, in the limit $N_{c} \rightarrow \infty$ [9]. Dynamical justifications of the rule of discarding $1 / N_{c}$ contributions have been investigated by analyzing the sign and the size of nonfactorizable matrix elements in some $D$ and $B$ meson nonleptonic decays 10,11]. 
However, in order to have a quantitative overview of the validity of the factorization approach we need a careful determination, either from experiment or by a QCD calculation, of the form factors appearing in the factorized amplitudes like (1.12). This is an important preliminary study in the analysis of the decay channels where naive factorization seems to fail [12].

Early estimates of $F_{1}, V, A_{1}$ and $A_{2}$, evaluated at $q^{2}=0$ or at $q^{2}=q_{\max }^{2}$, are available from constituent quark models [7, 13, 14]. More recently, these form factors have been computed, at a fixed value of $q^{2}$, by QCD sum rules [15] 17] and by lattice QCD [18]; they have also been derived in the framework of models that incorporate heavy quark and chiral symmetries [19]. Moreover, QCD sum rules have been used to determine the $q^{2}$ dependence of $F_{1}, V, A_{1}, A_{2}$ and $F_{0}$ [15 [17,20,21]; data on this functional dependence are also available from lattice QCD, but with large error bars [18.

As for $A_{0}\left(q^{2}\right)$, the procedure adopted so far consists in using eqs. $1.10-1.11$ ) to get $A_{0}(0)$ from $A_{1}(0)$ and $A_{2}(0)$, and then in assuming a suitable dependence on $q^{2}$, invoking the dominance of the nearest pole in the $t=q^{2}$ channel.

This procedure has two difficulties. The first one is that $A_{1}(0)$ and $A_{2}(0)$ are predicted by QCD sum rules and lattice QCD in a range of values; the uncertainty (larger for $A_{2}$ ) is determined by the variation of the parameters employed in the calculation (which are not accurately known) and by the statistical error in lattice simulations. This implies that $A_{0}(0)$ is determined with a large error (within an order of magnitude for the transition $B \rightarrow \rho$ ), an uncertainty that heavily affects the analysis of the accuracy of the factorization approach. This error is even more important in the study of light $S U(3)_{F}$ breaking effects, an argument which has recently prompted a number of interesting investigations [22].

The second point concerns the functional dependence of $A_{0}$ on $q^{2}$, which is needed in computing nonleptonic transitions. The assumption that, also for low values of $q^{2}$, the form factor $A_{0}$ is dominated by the nearest resonance requires an explicit check, since it is known that, for the transitions $B, D \rightarrow \rho, K^{*}$, the form factors $A_{1}$ and $A_{2}$ obtained by QCD sum rules appear to be nearly independent of $q^{2}$ up to rather large values of the squared momentum transferred 15, 16, 21].

Both these difficulties can be avoided by a direct calculation of $A_{0}\left(q^{2}\right)$. This is the problem we address in the present paper: we compute $A_{0}\left(q^{2}\right)$ for the transitions $B \rightarrow \rho$, $D \rightarrow \rho, K^{*}$ and $B \rightarrow D^{*}$ using three-point function QCD sum rules. In Sect.2 we derive the sum rule for $A_{0}\left(q^{2}\right)$, and in Sect.3 we collect our numerical results, together with a comparison with the outcome of other calculations.

The last point we analyze in this note is a proposal, put forward in ref. 223 25], to relate the branching ratio of the rare transition $B \rightarrow K^{*} \gamma$ to the spectrum of the semileptonic decay $B \rightarrow \rho \ell \nu$. This connection could have interesting phenomenological consequences, e.g. for the measurement of $V_{u b}$; it is based on the equality, obtained in the infinite heavy quark mass limit, $T_{1}(0)=A_{0}^{B \rightarrow \rho}(0)$, where $T_{1}$ is the form factor governing $B \rightarrow K^{*} \gamma$. We shall discuss the validity of this relation and show that there is a sizeable deviation to be taken into account. 


\section{THE FORM FACTOR $A_{0}$ FROM THREE POINT FUNCTION QCD SUM RULES.}

In order to calculate the form factor $A_{0}\left(q^{2}\right)$ in eq.(1.8) using three-point sum rules, let us consider the correlator [26,27]

$$
T_{\mu \nu}^{D \rightarrow K^{*}}\left(p, p^{\prime}, q\right)=i^{2} \int d x d y e^{i\left(p^{\prime} \cdot x-p \cdot y\right)}<0\left|T\left\{j_{\nu}(x) A_{\mu}(0) j_{5}^{\dagger}(y)\right\}\right| 0>.
$$

Here the weak current $A_{\mu}=\bar{s} \gamma_{\mu} \gamma_{5} c$ represents the flavour changing axial current in (1.8), while the quark currents $j_{\nu}=\bar{d} \gamma_{\nu} s$ and $j_{5}=\bar{d} i \gamma_{5} c$ interpolate $K^{*}$ and $D$ mesons, respectively, and have a non-vanishing matrix element between the vacuum and $K^{*}$ and $D$ states: eq.(1.6) and

$$
<0\left|j_{5}\right| D(p)>=f_{D} \frac{m_{D}^{2}}{m_{c}}
$$

where $m_{c}$ is the mass of the charm quark (hereafter we put the masses of the light $u$ and $d$ quarks to zero). The product $q^{\mu} T_{\mu \nu}$ can be decomposed in two independent Lorentz structures:

$$
q^{\mu} T_{\mu \nu}=-i\left[P_{\nu} T_{1}\left(p^{2}, p^{\prime 2}, q^{2}\right)+q_{\nu} T_{2}\left(p^{2}, p^{2}, q^{2}\right)\right]
$$

with $P_{\nu}=\left(p+p^{\prime}\right)_{\nu}$, and the two scalar functions $T_{i}(i=1,2)$ can be represented by a double dispersion relation:

$$
T_{i}\left(p^{2}, p^{\prime 2}, q^{2}\right)=\frac{1}{(2 \pi)^{2}} \int d s d s^{\prime} \frac{\rho_{i}^{(h a d)}\left(s, s^{\prime}, q^{2}\right)}{\left(s-p^{2}\right)\left(s^{\prime}-p^{\prime 2}\right)}+\text { subtractions }
$$

with the spectral functions $\rho_{i}^{(h a d)}$ expressed in terms of physical states:

$$
\rho_{i}^{(h a d)}=\rho_{i}^{(r e s)}+\rho_{i}^{(h r)}
$$

In (2.5) $\rho_{i}^{\text {(res) }}$ represent the contribution of the lowest lying resonances (in the zero width approximation) and are expressed in terms of the form factor $A_{0}^{D \rightarrow K^{*}}$ we are interested in:

$$
\begin{aligned}
& \rho_{1}^{(r e s)}\left(s, s^{\prime}, q^{2}\right)=(2 \pi)^{2} f_{K^{*}} f_{D} \frac{m_{D}^{2}}{2 m_{c}}\left(m_{K^{*}}^{2}-m_{D}^{2}+q^{2}\right) A_{0}^{D \rightarrow K^{*}}\left(q^{2}\right) \delta\left(s-m_{D}^{2}\right) \delta\left(s^{\prime}-m_{K^{*}}^{2}\right) \\
& \rho_{2}^{(r e s)}\left(s, s^{\prime}, q^{2}\right)=(2 \pi)^{2} f_{K^{*}} f_{D} \frac{m_{D}^{2}}{2 m_{c}}\left(3 m_{K^{*}}^{2}+m_{D}^{2}-q^{2}\right) A_{0}^{D \rightarrow K^{*}}\left(q^{2}\right) \delta\left(s-m_{D}^{2}\right) \delta\left(s^{\prime}-m_{K^{*}}^{2}\right)
\end{aligned}
$$

while $\rho_{i}^{(h r)}$ take contribution from higher states and from the hadronic continuum.

The correlator (2.1) can also be calculated in QCD by the operator product expansion $(\mathrm{OPE})$ in the region of large Euclidean momenta $p^{2}, p^{2}$. In this expansion the most singular term is represented by the perturbative term, which can be obtained evaluating a triangle diagram. It has a dispersive representation similar to (2.4): 


$$
T_{i}^{(p e r t)}\left(p^{2}, p^{\prime 2}, q^{2}\right)=\frac{1}{(2 \pi)^{2}} \int d s d s^{\prime} \frac{\rho_{i}^{(p e r t)}\left(s, s^{\prime}, q^{2}\right)}{\left(s-p^{2}\right)\left(s^{\prime}-p^{\prime 2}\right)}
$$

the explicit form of the spectral functions $\rho_{i}^{(p e r t)}$ can be found in the appendix. The next terms in the OPE can be read as:

$$
T_{i}^{(n p)}=\sum_{n} d_{i}^{(n)}\left(p^{2}, p^{2}, q^{2}\right)<0\left|O_{n}\right| 0>
$$

where the local operators $O_{n}$, written in terms of quark and gluon fields, are ordered according to their increasing dimension; their vacuum matrix elements (condensates) parametrize the effects of the nonperturbative QCD vacuum. Here we only consider the contribution of the operators of dimension $D=3$ and $D=5$ :

$$
T_{i}^{(n p)}=d_{i}^{(3)}<\bar{q} q>+d_{i}^{(5)}<\bar{q} g_{s} \sigma G q>
$$

$<\bar{q} q>$ and $<\bar{q} g_{s} \sigma G q>$ are the quark and the mixed quark-gluon condensates, respectively; the coefficients $d_{i}^{(3)}$ and $d_{i}^{(5)}$ can be calculated in perturbation theory.

The hadronic and the QCD representations of the correlator (2.1) can be used to derive two sum rules for $A_{0}\left(q^{2}\right)$. As a matter of fact, we invoke quark-hadron duality and assume the equality:

$$
\int_{D^{\prime}} d s d s^{\prime} \frac{\rho_{i}^{(h r)}\left(s, s^{\prime}, q^{2}\right)}{\left(s-p^{2}\right)\left(s^{\prime}-p^{\prime 2}\right)}=\int_{D^{\prime}} d s d s^{\prime} \frac{\rho_{i}^{(p e r t)}\left(s, s^{\prime}, q^{2}\right)}{\left(s-p^{2}\right)\left(s^{\prime}-p^{\prime 2}\right)}
$$

in a region $D^{\prime}$ above some threshold $s_{0}, s_{0}^{\prime}$. This assumption can be realized if we adopt the model:

$$
\rho_{i}^{(h r)}\left(s, s^{\prime}, q^{2}\right)=\rho_{i}^{(p e r t)}\left(s, s^{\prime}, q^{2}\right)\left(1-\Theta\left(s_{0}-s\right) \Theta\left(s_{0}^{\prime}-s^{\prime}\right)\right) .
$$

Using (2.12) the following sum rules for $A_{0}\left(q^{2}\right)$ can be written:

$$
\begin{aligned}
\frac{1}{(2 \pi)^{2}} \int_{D} d s d s^{\prime} \frac{\rho_{i}^{(\text {res })}\left(s, s^{\prime}, q^{2}\right)}{\left(s-p^{2}\right)\left(s^{\prime}-p^{\prime 2}\right)} & =\frac{1}{(2 \pi)^{2}} \int_{D} d s d s^{\prime} \frac{\rho_{i}^{(\text {pert })}\left(s, s^{\prime}, q^{2}\right)}{\left(s-p^{2}\right)\left(s^{\prime}-p^{\prime 2}\right)} \\
& +d_{i}^{(3)}<\bar{q} q>+d_{i}^{(5)}<\bar{q} g_{s} \sigma G q>
\end{aligned}
$$

where the region $D$ is fixed by:

$$
m_{c}^{2} \leq s \leq s_{0}
$$

and

$$
\begin{gathered}
m_{s}^{2} \leq s^{\prime} \leq s_{0}^{\prime} \\
s_{-}^{\prime}\left(s, q^{2}\right) \leq s^{\prime} \leq s_{+}^{\prime}\left(s, q^{2}\right)
\end{gathered}
$$

with ( $m_{s}$ is the strange quark mass): 


$$
\begin{aligned}
s_{ \pm}^{\prime}\left(s, q^{2}\right) & =\frac{1}{2}\left\{\left(s-m_{c}^{2}\right)\left(1-\frac{q^{2}}{m_{c}^{2}}\right)+\left(\frac{m_{s}}{m_{c}}\right)^{2}\left(s+m_{c}^{2}\right)\right. \\
& \left. \pm\left(s-m_{c}^{2}\right)\left[\left(\frac{m_{s}}{m_{c}}\right)^{4}+\left(1-\frac{q^{2}}{m_{c}^{2}}\right)^{2}-2\left(\frac{2 m_{s}^{2}}{m_{c}^{2}}\right)\left(1+\frac{q^{2}}{m_{c}^{2}}\right)\right]^{1 / 2}\right\}
\end{aligned}
$$

In (2.13) we have omitted the subtraction terms, that can still be present. They are removed by performing a double Borel transform to both sides of (2.13) in the variables $P^{2}=-p^{2}$ and $P^{\prime 2}=-p^{\prime 2}$ :

$$
B_{P^{2}, P^{\prime 2}}\left(M^{2}, M^{\prime 2}\right)=\left[\frac{1}{n !}\left(-P^{2}\right)^{n+1}\left(\frac{d}{d P^{2}}\right)^{n+1}\right]\left[\frac{1}{m !}\left(-P^{\prime 2}\right)^{m+1}\left(\frac{d}{d P^{\prime 2}}\right)^{m+1}\right]
$$

in the limit $P^{2}, P^{\prime 2} \rightarrow \infty, n, m \rightarrow \infty, M^{2}=P^{2} / n M^{\prime 2}=P^{\prime 2} / m$ fixed. The resulting sum rules for $A_{0}^{D \rightarrow K^{*}}$ read:

$$
\begin{aligned}
A_{0}^{D \rightarrow K^{*}}\left(q^{2}\right) & =\frac{H_{i}}{(2 \pi)^{2}} \int_{D} d s d s^{\prime} \rho_{i}^{(p e r t)}\left(s, s^{\prime}, q^{2}\right) \exp \left(-\frac{\left(s-m_{D}^{2}\right)}{M^{2}}-\frac{\left(s^{\prime}-m_{K^{*}}^{2}\right)}{M^{\prime 2}}\right) \\
& +\left[\Gamma_{i}^{D=3}<\bar{q} q>+\Gamma_{i}^{D=5}<\bar{q} g_{s} \sigma^{\mu \nu} G_{\mu \nu}^{a} \frac{\lambda^{a}}{2} q>\right] \exp \left(\frac{m_{D}^{2}-m_{c}^{2}}{M^{2}}+\frac{m_{K^{*}}^{2}-m_{s}^{2}}{M^{\prime 2}}\right)
\end{aligned}
$$

$(i=1,2)$; the coefficients $H_{i}, \Gamma_{i}^{D=3}$ and $\Gamma_{i}^{D=5}$ are collected in the appendix.

Eq.(2.18) represents two independent sum rules for $A_{0}^{D \rightarrow K^{*}}\left(q^{2}\right)$, that will be analyzed in the next section. Now, before discussing the parameters and the criteria we have used to compute $A_{0}^{D \rightarrow K^{*}}$, let us consider the $t$ dependence in (2.18). In principle, the operator product expansion can be reliably applied to the evaluation of the correlator (2.1) in the region af large euclidean values of $q^{2}$. However, as discussed in [15], we can also consider positive values of $q^{2}$ provided that the occurrence of non-Landau singularities either is avoided or is carefully taken into account. Such singularities remain far from the integration region of the dispersive integral if $q^{2}$ is small with respect to the physical threshold in the $t$ channel: $t_{t h}=\left(m_{c}+m_{s}\right)^{2}$; their presence is shown up by the appearance of branch points (for large and positive $q^{2}$ ) in the spectral functions $\rho_{i}^{(\text {pert })}$.

Taking into account the above considerations, we study the form factor $A_{0}$ using eq.(2.18) for small positive values of $q^{2}$, and not in the whole physically accessible $t$-region. However, the information we obtain on the $t$-dependence of the form factor is enough to describe a large number of nonleptonic $D(B)$-meson decays. 


\section{NUMERICAL ANALYSIS OF THE SUM RULES}

The numerical analysis of the sum rules (2.18) is performed using the following values for the quark condensates, taken at a low renormalization scale $(\mu \simeq 1 \mathrm{GeV})$ [26]:

$$
\begin{aligned}
<\bar{q} q> & =(-230 M e V)^{3} \\
<\bar{q} g_{s} \sigma^{\mu \nu} G_{\mu \nu}^{a} \frac{\lambda^{a}}{2} q> & =m_{0}^{2}<\bar{q} q>
\end{aligned}
$$

with $m_{0}^{2}=0.8 \mathrm{GeV}^{2}$. The condensates can be evaluated at higher scales using the leadinglog approximation for the anomalous dimension of the quark and of the mixed quark-gluon condensates; however, this rescaling does not affect sensitively the numerical result for $A_{0}$.

As for the quark masses, we use: $m_{c}=1.35 \mathrm{GeV}$ and $m_{s}=0.16 \mathrm{GeV}$; moreover, we use $m_{b}=4.6 \mathrm{GeV}$ in the calculation of form factor connected with the transition $B \rightarrow \rho$. The leptonic constant $f_{K^{*}}$ can be derived from the measurement of the branching ratio $\tau \rightarrow K^{*} \nu_{\tau}: f_{K^{*}}=0.22 \pm 0.01 \mathrm{GeV}$; we use $f_{D}=195 \pm 20 \mathrm{MeV}$ for the leptonic constant of the $D$ meson.

A comment on this value of $f_{D}$ is in order. This value comes from two-point QCD sum rules, including radiative corrections at the order $\mathcal{O}\left(\alpha_{s}\right)$ [28,29]; these corrections are at level of $13-15 \%$ for $f_{D}, f_{D^{*}}$ and play a major role in $f_{B}$; including the corrections the results are in agreement with lattice QCD. On the other hand, the QCD expression of the three-point correlator has been computed at zero order in $\alpha_{s}$. Several authors [15, when computing the form factors, adopt the strategy of taking the ratio of three and two-point functions, calculated at the same order in $\alpha_{s}$ in order to be consistent, trying to reduce the uncertainty deriving from higher order contributions. However, the sign of the radiative corrections to three point functions is not known on general grounds; for this reason our attitude is to use the best known values of the leptonic constants, e.g. the values on which QCD sum rules and lattice QCD agree.

The effective threshold $s_{0}$ and $s_{0}^{\prime}$ must be chosen in a range of values between the mass squared of the lowest lying resonances and the first excited states. They have been fixed by studying two-point sum rules, for the calculation of static properties as the mass of the leptonic constant of the particles, and three-point functions sum rules in the calculation of the semileptonic $D \rightarrow K^{*} \ell \nu$ decay [15: $s_{0}=7-8 \mathrm{GeV}^{2}$ for the channel of $D$ meson, and

$s_{0}^{\prime}=1.5-1.7 \mathrm{GeV}^{2}$ for the channel of $K^{*}$. The variation of the values of $s_{0}, s_{0}^{\prime}$ induces a variation of the predicted form factor $A_{0}$ that can be considered a theoretical uncertainty in the final result.

Using the parameters above, $A_{0}^{D \rightarrow K^{*}}\left(q^{2}\right)$ can be obtained from (2.18) as a function of the Borel parameters $M^{2}$ and $M^{\prime 2}$. However, since these variables are unphysical, we search a region in $M^{2}, M^{\prime 2}$ where $A_{0}$ does not depend on them (stability plateau). In this region other conditions must be verified. The first constraint consists in checking a hierarchical structure of the various terms of (2.18):

$$
T^{(\text {pert })} \geq T^{(D=3)} \geq T^{(D=5)} ;
$$

only if the OPE displays this structure we can hope that higher order power corrections in (2.18) can be safely neglected. 
Another condition is connected with the approximation (2.11). Since we are not guaranteed that quark-hadron duality starts already in correspondence to the first hadronic excitations, we must choose $M^{2}, M^{\prime 2}$ small enough to enhance the contribution of the lowest lying states in the sum rule, and to exponentially suppress the contribution of the continuum. We check this condition by verifying that

$$
\left|\int_{D} d s d s^{\prime} \rho_{i}^{(p e r t)}\left(s, s^{\prime}, q^{2}\right) e^{-\frac{\left(s-m_{D}^{2}\right)}{M^{2}}-\frac{\left(s^{\prime}-m_{K^{*}}^{2}\right)}{M^{\prime 2}}}\right| \geq \alpha\left|\int_{D_{1}} d s d s^{\prime} \rho_{i}^{(p e r t)}\left(s, s^{\prime}, q^{2}\right) e^{-\frac{\left(s-m_{D}^{2}\right)}{M^{2}}-\frac{\left(s^{\prime}-m_{K^{*}}^{2}\right)}{M^{\prime 2}}}\right|
$$

where $D_{1}$ is an integration region larger than $D$ (it extends to $10 s_{0}$ and $10 s_{0}^{\prime}$ ) and $\alpha=$ $0.4-0.5$. From the condition (3.3) an upper bound to $M^{2}, M^{\prime 2}$ can be determined.

In principle both the sum rules $(i=1,2)$ in $(2.18)$ can be used to calculate $A_{0}$. However, it turns out that only for the first sum rule $(i=1)$ the condition (3.3) is fulfilled. In the second sum rule the constraint (3.3) is never verified as a consequence, probably, of the major role played by higher states. For this reason we exclude the second sum rule in our analysis and use only the first one to determine $A_{0}$.

Let us consider the form factor $A_{0}^{D \rightarrow K^{*}}\left(q^{2}\right)$. Our results, obtained for $M^{2}=3 \mathrm{GeV}^{2}$ and $M^{\prime 2}=1.5 \mathrm{GeV}^{2}$, are depicted in fig.(1) for several sets of parameters. At $q^{2}=0$ we have: $A_{0}^{D \rightarrow K^{*}}(0)=0.58 \pm 0.05$, where the uncertainty comes from the variation of the parameters. For positive values of $q^{2}$ we observe an increasing of the form factor; if we use the simple pole formula:

$$
A_{0}^{D \rightarrow K^{*}}\left(q^{2}\right)=\frac{A_{0}^{D \rightarrow K^{*}}(0)}{1-q^{2} / m_{P}^{2}}
$$

to fit the $q^{2}$ dependence, we obtain that the fitted pole mass is: $m_{P}=1.65 \mathrm{GeV}$, which is not far from the experimental mass of the particle having the same quantum numbers of the pole: $m_{D_{s}}=1.969 \mathrm{GeV}$.

Let us now consider the transition $D \rightarrow \rho$. The constant $f_{\rho^{+}}$, computed from the decay $\rho^{0} \rightarrow e^{+} e^{-}$and assuming the isospin symmetry, is given by: $f_{\rho^{+}}=216 \mathrm{MeV}$. Using $s_{0}^{\prime}=1.3-1.5 \mathrm{GeV}^{2}$ we get: $A_{0}^{D \rightarrow \rho}(0)=0.52 \pm 0.05$. The fitted pole mass is $m_{P}=1.6 \mathrm{GeV}$, to be compared with $m_{D}=1.865 \mathrm{GeV}$ which is the mass of the nearest pole in the $t$ channel (fig.(2)).

From the above results we would get: $r=A_{0}^{D \rightarrow K^{*}}(0) / A_{0}^{D \rightarrow \rho}(0)=1.12 \pm 0.11$; however, the light $S U(3)_{F}$ breaking effects can be better estimated by studying the ratio of the sum rules for the transitions $D \rightarrow K^{*}$ and $D \rightarrow \rho$, which is stable with respect to the variation of the input parameters. We get $r=1.10 \pm 0.05$, i.e. an $S U(3)_{F}$ breaking effect at the level of $10 \%$.

We have also computed the form factor $A_{0}^{B \rightarrow \rho}$ appearing in nonleptonic $B$ meson decays which are interesting due to their connection to the measurement of $V_{u b}$; using $s_{0}=33-$ $36 \mathrm{GeV}^{2}$ and $f_{B}=180 \pm 20 \mathrm{MeV}$ as obtained by two point function QCD sum rules, we get $A_{0}^{B \rightarrow \rho}(0)=0.24 \pm 0.02$ and a pole mass $m_{P}=5 \mathrm{GeV}$, to be compared to $m_{B}=5.275 \mathrm{GeV}$; the form factor is depicted in fig.(3) in the range $q^{2}=0-10 \mathrm{GeV}^{2}$.

Finally, for the heavy-to-heavy meson transition $B \rightarrow D^{*}$ we get (using $f_{D^{*}}=250 \mathrm{MeV}$ 28]): $A_{0}^{B \rightarrow D^{*}}(0)=0.65 \pm 0.05$. 
Let us now compare our results with existing predictions for $A_{0}(0)$, obtained from the values of $A_{1}(0)$ and $A_{2}(0)$ in the corresponding channels (see Table I). The first observation is that our predictions appear to be smaller than from potential models [7, 13, 14] (the exception is $A_{0}^{B \rightarrow \rho}$ in 13$]$ ). On the other hand, the $S U(3)$ breaking effects are of the same size as in our approach.

The comparison with QCD sum rules predictions [15,16] shows the problem we have mentioned in the introduction: the uncertainties in the results obtained using $A_{1}(0)$ and $A_{2}(0)$ are so large that they obscure the real value of $A_{0}(0)$, whereas the error in our predictions is at the level of $10 \%$, and therefore a substantial improvement has been obtained.

It is interesting to compare our predictions to the outcome of [19] obtained in a framework based on heavy-quark and chiral symmetries, using as an input the experimental data on $D \rightarrow K^{*} \ell \nu$. Although there is a remarkable agreement on $D \rightarrow K^{*}$ and $B \rightarrow \rho$, the light $S U(3)_{F}$ breaking corrections connecting $D \rightarrow K^{*}$ to $D \rightarrow \rho$ are of similar size than in our approach but with opposite sign.

The last point we would like to mention is that, in our calculation, the form factors $A_{0}$ in the different channels increase for positive values of $q^{2}$, and that their functional dependence is compatible with the simple pole behaviour dominated by the nearest resonance in the $t$ channel.

\section{IV. $B \rightarrow K^{*} \gamma$ VERSUS $B \rightarrow \rho \ell \nu$}

In this section we discuss a procedure, proposed in refs. [23,24], to relate the width of the radiative $B \rightarrow K^{*} \gamma$ transition to the spectrum of the Cabibbo suppressed semileptonic decay $B \rightarrow \rho \ell \nu$. This relation can be used to constrain the electroweak parameters involved in these processes and to reduce their dependence on the models for the hadronic form factors.

The radiative rare $B$ meson decays, like $B \rightarrow K_{i}^{*} \gamma\left(K_{i}^{*}=K^{*}(890), K_{1}(1400)\right.$, etc. $)$, have been extensively studied, from the theoretical standpoint, since they have a peculiar role in the precision tests of the quark sector in the Standard Model (SM) and probe the effects of new physics beyond the Standard Model [30]. Within SM they are induced by the one-loop electromagnetic penguin operator $b \rightarrow s \gamma\left(\right.$ for $\left.m_{s}<<m_{b}\right)$ [31:

$$
\mathcal{H}_{e f f}(b \rightarrow s)=\frac{G_{F}}{\sqrt{2}} C m_{b} \epsilon^{\mu} \bar{s} \sigma_{\mu \nu} \frac{1+\gamma_{5}}{2} q^{\nu} b
$$

where the constant $C$ contains the dependence on the Cabibbo-Kobayashi-Maskawa matrix elements, on the QCD correcting terms and on the ratio $m_{t o p} / m_{W}$ (the explicit formulae can be found in ref. [31].

The description of the exclusive decays requires the knowledge of form factors. For example, the relevant matrix element for the transition $B \rightarrow K^{*}(890) \gamma$ can be written as follows: 


$$
\begin{aligned}
\left\langle K^{*}\left(p^{\prime}, \lambda\right)\left|\bar{s} \sigma_{\mu \nu} q^{\nu} \frac{\left(1+\gamma_{5}\right)}{2} b\right| B(p)\right\rangle= & i \varepsilon_{\mu \nu \rho \sigma} \epsilon^{* \nu} p^{\rho} p^{\prime \sigma} T_{1}\left(q^{2}\right) \\
& +\left[\left(m_{B}^{2}-m_{K^{*}}^{2}\right) \epsilon_{\mu}^{*}-\left(\epsilon^{*} \cdot q\right)\left(p+p^{\prime}\right)_{\mu}\right] T_{2}\left(q^{2}\right) \\
& +\left(\epsilon^{*} \cdot q\right)\left[\left(p-p^{\prime}\right)_{\mu}-\frac{q^{2}}{\left(m_{B}^{2}-m_{K^{*}}^{2}\right)}\left(p+p^{\prime}\right)_{\mu}\right] T_{3}\left(q^{2}\right) .
\end{aligned}
$$

The form factors $T_{i}\left(q^{2}\right)$ require a nonperturbative evaluation. However, in the static limit for the $b$ quark and using the $S U(3)_{F}$ symmetry for the light quarks, a number of interesting relations can be derived between $T_{i}$ and the form factors $V$ and $A_{i}$ governing $B \rightarrow \rho \ell \nu$ 23,32]. Although such relations are obtained at zero recoil $\left(q_{\text {max }}^{2}=\left(m_{B}-m_{K^{*}, \rho}\right)^{2}\right)$, it has been argued that they continue to hold in the whole physically accessible $q^{2}$ range [23, 33, ,34], and therefore that one is able to relate the width of $B \rightarrow K^{*} \gamma$ to observable quantities in $B \rightarrow \rho \ell \nu$. An example is represented by the ratio:

$$
\Gamma\left(B \rightarrow K^{*} \gamma\right)\left(\lim _{q^{2} \rightarrow 0, \text { curve }} \frac{1}{q^{2}} \frac{d \Gamma(B \rightarrow \rho e \bar{\nu})}{d E_{\rho} d E_{e}}\right)^{-1}=\frac{4 \pi^{2}}{G_{F}^{2}} \frac{|\eta|^{2}}{\left|V_{u b}\right|^{2}} \frac{\left(m_{B}^{2}-m_{K^{*}}^{2}\right)^{3}}{m_{B}^{4}}
$$

that Burdman and Donoghue [23] predict to be independent of any form factor. In (4.3) curve means the region in the Dalitz plot where $q^{2}=4 E_{e}\left(m_{B}-E_{\rho}-E_{e}\right)$; the factor $\eta$ includes the $Q C D$ corrections to the $b \rightarrow s \gamma$ decay and the other relevant electroweak parameters [23].

Eq.(4.3) is interesting, both from the experimental and the theoretical point of view, since it would provide, for example, a method to measure $V_{u b}$ without referring to calculations of the form factors, from the measurement $B R\left(B \rightarrow K^{*} \gamma\right)=(4.5 \pm 1.0 \pm 0.9) \times 10^{-5}$ [35] and the spectrum of $B \rightarrow \rho \ell \nu$. The drawback, pointed out by O'Donnell and Tung [24], is that the semileptonic decay spectrum vanishes at $q^{2}=0$ on this curve, with the consequence that the number of experimental points required to measure the denominator in (4.3) could be insufficient for a reliable determination of $V_{u b}$.

A more convenient observable [25,36] is represented by the ratio:

$$
R\left(B \rightarrow K^{*} \gamma\right)\left(\left.\frac{d \Gamma\left(\bar{B} \rightarrow \rho l \bar{\nu}_{l}\right)}{d q^{2}}\right|_{q^{2}=0}\right)^{-1}=\frac{192 \pi^{3}}{G_{F}^{2}} \frac{1}{\left|V_{b u}\right|^{2}} \frac{\left(m_{B}^{2}-m_{K^{*}}^{2}\right)^{3}}{\left(m_{B}^{2}-m_{\rho}^{2}\right)^{3}} \frac{m_{b}^{3}}{\left(m_{b}^{2}-m_{s}^{2}\right)^{3}}|\mathcal{I}|^{2}
$$

In (4.4) $R\left(B \rightarrow K^{*} \gamma\right)=\Gamma\left(B \rightarrow K^{*} \gamma\right) / \Gamma(b \rightarrow s \gamma)$ is independent of the electroweak parameters appearing in the effective Hamiltonian, and only depends on $T_{1}(0)$ :

$$
R\left(B \rightarrow K^{*} \gamma\right)=\frac{m_{b}^{3}\left(m_{B}^{2}-m_{K^{*}}^{2}\right)^{3}}{m_{B}^{3}\left(m_{b}^{2}-m_{s}^{2}\right)^{3}}\left|T_{1}(0)\right|^{2},
$$

having used the relation $T_{2}(0)=\frac{1}{2} T_{1}(0)$. The factor $\mathcal{I}$ is given by [25]:

$$
\mathcal{I}=\frac{\left(m_{B}+m_{\rho}\right)}{\left(m_{B}+m_{K^{*}}\right)} \frac{T_{1}(0)}{A_{0}^{B \rightarrow \rho}(0)} .
$$

The main reason for studying the ratio (4.4) is that $d \Gamma\left(\bar{B} \rightarrow \rho l \bar{\nu}_{l}\right) / d q^{2}$ in (4.4) does not vanish at $q^{2}=0$. Moreover, the factor $\mathcal{I}$ should be close to one. As a matter of fact, in the 
framework of the Bauer-Stech-Wirbel model [0] the value $\mathcal{I}=1.12$ can be derived, whereas the constituent quark model 37 provides the value $\mathcal{I}=1.09-1.18$. The relation $\mathcal{I}=1$ has been obtained by the authors in ref. [24] by considering the transition $b \bar{q} \rightarrow Q \bar{q}$ in the limit where both the $b$ and $Q$ quarks are heavy, applying spin symmetry relations; then, it has been argued that this relation still holds for a light $Q$ quark in the weak binding limit for the meson.

The factor $\mathcal{I}$ can be derived by three point QCD sum rules, using realistic values of the quark masses in a fully relativistic approach. Using the determination $T_{1}(0)=0.35 \pm 0.05$ [33] and the value $A_{0}^{B \rightarrow \rho}(0)$ derived in the previous section, we get: $\mathcal{I}=1.43 \pm 0.32$. Notice that, in evaluating the error for $\mathcal{I}$, we have taken into account the correlated errors in the numerator and in the denominator of $\mathcal{I}$.

The uncertainty can be reduced by studying the ratio of the sum rules determining

$T_{1}(0)$ (the formulae can be found in [33]) and $A_{0}^{B \rightarrow \rho}(0)$. We find a wide stability window in correspondence to the result: $\mathcal{I}=1.3 \pm 0.1$.

Therefore, the QCD sum rules prediction differs by $10-30 \%$ from the values derived in [24,25]; this deviation still is model dependent, in the sense that it comes out from an explicit QCD sum rules calculation and not from symmetry arguments, and therefore it should be checked in a different approach, e.g. by lattice QCD.

In any case, we feel that, modulo this uncertainty on the value of $\mathcal{I}$, the idea of relating $B \rightarrow K^{*} \gamma$ to $B \rightarrow \rho \ell \nu$ provides us with an interesting alternative method to measure $V_{u b}$.

\section{CONCLUSIONS}

We have applied three-point function QCD sum rules to calculate the form factor $A_{0}$ appearing in the matrix element of the flavour changing axial current between the $D$ and $B$ mesons and a vector meson state. This form factor plays an important role in the calculation of non leptonic $B$ and $D$ decays to $P V$ states in the hypothesis of factorization.

The explicit calculation has allowed us to determine $A_{0}$ at zero momentum transferred, with better accuracy than in the indirect determination from $A_{1}$ and $A_{2}$. We have also computed the $q^{2}$ dependence, finding an increase for positive values of the squared momentum transferred, compatible with a simple polar behaviour.

Finally, we have considered the role of $A_{0}$ in relating the rare $B \rightarrow K^{*} \gamma$ decay with the semileptonic $B \rightarrow \rho \ell \nu$ spectrum, in the search of methods for measuring the matrix element $V_{u b}$.

\section{ACKNOWLEDGMENTS}

We thank F.Buccella, G.Nardulli and N.Paver for discussions. 


\section{APPENDIX A: PERTURBATIVE AND NON-PERTURBATIVE TERMS.}

The perturbative spectral functions $\rho_{i}^{(p e r t)}\left(s, s^{\prime}, q^{2}\right)$ in (2.8), at the lowest order in $\alpha_{s}$, can be obtained by the Cutkosky rules applied to a triangle diagram:

$$
\begin{aligned}
\rho_{1}\left(s, s^{\prime}, q^{2}\right) & =\frac{3}{4 \sqrt{\lambda}}\left\{\left(m_{c}+m_{s}\right)\left(\Delta^{\prime}-\Delta\right)+\frac{2\left(\Delta s^{\prime}+\Delta^{\prime} s\right)-u\left(\Delta^{\prime}+\Delta\right)}{\lambda}\right. \\
& \left.\times\left[-\left(m_{c}+m_{s}\right)\left(s-s^{\prime}+q^{2}\right)+2 m_{c}\left(m_{c}^{2}-m_{s}^{2}\right)\right]\right\}, \\
\rho_{2}\left(s, s^{\prime}, q^{2}\right) & =\frac{3}{4 \sqrt{\lambda}}\left\{\left(m_{c}+m_{s}\right)\left(\Delta^{\prime}+\Delta\right)+\frac{2\left(\Delta s^{\prime}-\Delta^{\prime} s\right)+u\left(\Delta-\Delta^{\prime}\right)}{\lambda}\right. \\
& \left.\times\left[\left(m_{c}+m_{s}\right)\left(-s+s^{\prime}-q^{2}\right)+2 m_{c}\left(m_{c}^{2}-m_{s}^{2}\right)\right]\right\},
\end{aligned}
$$

where $\Delta=s-m_{c}^{2}, \Delta^{\prime}=s^{\prime}-m_{s}^{2}, u=s+s^{\prime}-q^{2}$ and $\lambda=u^{2}-4 s s^{\prime}$.

The Borel transformed coefficients of the non-perturbative $D=3$ terms read:

$$
\begin{gathered}
\Gamma_{1}^{(D=3)}=\frac{m_{c}^{2}-m_{s}^{2}}{2} \\
\Gamma_{2}^{(D=3)}=-\frac{\left(m_{c}+m_{s}\right)^{2}}{2} ;
\end{gathered}
$$

the coefficients of the condensate of dimension $D=5$ terms are:

$$
\begin{aligned}
\Gamma_{1}^{(D=5)} & =\frac{m_{c}^{2}\left(m_{s}^{2}-m_{c}^{2}\right)}{8 M^{4}}+\frac{m_{s}^{2}\left(m_{s}^{2}-m_{c}^{2}\right)}{8 M^{\prime 4}}+\frac{\left(m_{c}^{2}-m_{s}^{2}\right)}{6 M^{2}}+\frac{\left(m_{c}^{2}-m_{s}^{2}\right)}{12 M^{\prime 2}} \\
& +\frac{\left(m_{s}^{2}-m_{c}^{2}\right)\left(2 m_{s}^{2}-m_{c}^{2}\right)-q^{2}\left(m_{s}+m_{c}\right)\left(2 m_{s}-m_{c}\right)}{12 M^{2} M^{\prime 2}}
\end{aligned}
$$

and

$$
\begin{aligned}
\Gamma_{2}^{(D=5)} & =\frac{m_{c}^{2}\left(m_{s}+m_{c}\right)^{2}}{8 M^{4}}+\frac{m_{s}^{2}\left(m_{s}+m_{c}\right)^{2}}{8 M^{\prime} 4}-\frac{\left(m_{c}^{2}+3 m_{s}^{2}+4 m_{s} m_{c}\right)}{12 M^{\prime 2}}-\frac{\left(3 m_{c}^{2}+m_{s}^{2}+4 m_{s} m_{c}\right)}{6 M^{2}} \\
& +\frac{\left(m_{s}^{2}+m_{c}^{2}-q^{2}\right)\left(2 m_{s}^{2}+m_{c}^{2}\right)+m_{s} m_{c}\left(4 m_{c}^{2}+2 m_{s}^{2}-3 q^{2}\right)}{12 M^{2} M^{\prime 2}} .
\end{aligned}
$$

Finally, the coefficients $H_{i}$ in (2.18) read:

$$
H_{1}=\frac{2 m_{c}}{f_{K^{*}} f_{D} m_{D}^{2}\left(m_{K^{*}}^{2}-m_{D}^{2}+q^{2}\right)}
$$

and

$$
H_{2}=\frac{2 m_{c}}{f_{K^{*}} f_{D} m_{D}^{2}\left(3 m_{K^{*}}^{2}+m_{D}^{2}-q^{2}\right)} .
$$

The formulae for the transitions $D \rightarrow \rho, B \rightarrow \rho$ and $B \rightarrow D^{*}$ can be easily obtained. 


\section{REFERENCES}

[1] M.K.Gaillard and B.W.Lee, Phys. Rev. Lett. 33 , 108 (1974);

G.Altarelli and L.Maiani, Phys. Lett. B 52, 351 (1974).

[2] J.Schwinger, Phys. Rev. Lett. 12, 630 (1964).

[3] M.Bauer, B.Stech and M.Wirbel, Z. fur Phys. C 34, 103 (1987).

[4] F.Buccella, M.Lusignoli, G.Miele and A.Pugliese, Z. fur Phys. C 55, 243 (1992);

F.Buccella, M.Lusignoli, G.Mangano, G.Miele, A.Pugliese and P.Santorelli, Phys. Lett. B 302, 319 (1993);

A.Pugliese and P.Santorelli, Napoli preprint DSF-T-93/26; to appear in the Proceedings of the Third Workshop on the Tau-Charm Factory, J.Kirkby and R.Kirkby Eds., Editions Frontieres, 1994;

F.Buccella, M.Lusignoli, G.Miele, A.Pugliese and P.Santorelli, Preprint Roma n. 1026 and DSF-T-18/94.

[5] An example of the use of measured phase shifts in heavy meson physics (distance effects in $D^{0}-\bar{D}^{0}$ mixing) can be found in: P.Colangelo, G.Nardulli, N.Paver, Phys. Lett. B 242, $71(1990)$.

[6] G. Altarelli, G. Curci, G. Martinelli and S. Petrarca, Nucl. Phys. B 187, 461 (1981);

A.J. Buras, M. Jamin, M.E. Lautenbacher and P.E. Weisz, Nucl.Phys. B 370, 69 (1992) and Nucl.Phys. B 375, 501 (1992) (addendum);

M. Ciuchini, E. Franco, G. Martinelli and L. Reina, Nucl. Phys. B 415, 403 (1994).

[7] M.Wirbel, B.Stech and M.Bauer, Z. fur Phys. C 29, 637 (1985).

[8] A.J.Buras, J.M.Gerard and R.Ruckl, Nucl. Phys. B 268, 16 (1986).

[9] G. 't Hooft, Nucl. Phys. B 72, 461 (1974);

E. Witten, Nucl. Phys. B 149, 285 (1979).

[10] B.Block and M. A. Shifman, Sov. Journ. Nucl. Phys. 45, 135 (1987); 301 (1987); 522 (1987).

B.Block and M. A. Shifman, Nucl. Phys. B 389, 534 (1993).

[11] A.Khodjamirian and R.Ruckl, report MPI-PhT/94-26, May 1994.

[12] M.S.Alam et al. (CLEO Collab.), Preprint CLNS 94-1270, CLEO 94-5, hep-ph-9403295.

[13] F.G.Gilman and R.L.Singleton, Phys. Rev. D 41, 142 (1990).

[14] B.Grinstein, N.Isgur and M.B.Wise, Phys. Rev. Lett. 56, 298 (1986);

B.Grinstein, N.Isgur, D.Scora and M.B.Wise, Phys. Rev. D 39, 799 (1989);

N.Isgur and D.Scora, Phys. Rev. D 40, 1491 (1989).

[15] P.Ball, V.M.Braun and H.G.Dosh, Phys. Rev. D44, 3567 (1991).

[16] P.Ball, Phys. Rev. D48, 3190 (1993).

[17] V.M. Belyaev, A.Kodjamirian and R.Ruckl, Z. Phys. C60, 349 (1993).

[18] A.S.Abada et al., Nucl. Phys. B 416, 675 (1994).

[19] R.Casalbuoni, A.Deandrea, N.Di Bartolomeo, F. Feruglio, R.Gatto and G.Nardulli, Phys. Lett. B 299, 139 (1993).

[20] P.Colangelo and P.Santorelli, Phys. Lett. B 327, 123 (1994).

[21] For a review see: P.Colangelo, preprint Bari-Th/93-163, to appear in the Proceedings of the Third Workshop on the Tau-Charm Factory, J.Kirkby and R.Kirkby Eds., Editions Frontieres, 1994.

[22] See, for example: L.L.Chau and H.Y. Cheng, preprint UTP-SB-93-49, hep-ph/9404207.

[23] G.Burdman and J.F.Donoghue, Phys. Lett. B 270, 55 (1991). 
[24] P.J.O’Donnell and H.K.K.Tung, Phys. Rev. D 48, 2145 (1993).

[25] P.J.O'Donnell and Q.P.Xu, Phys. Lett. B 325, 219 (1994).

[26] M.A.Shifman, A.I.Vainshtein and V.I.Zakharov, Nucl. Phys. B 147, 385 (1979). For a review see the reprints volume: "Vacuum structure and QCD Sum Rules", M.A.Shifman, ed., North-Holland, 1992.

[27] B.L.Ioffe and A.V.Smilga, Nucl. Phys. B 216, 373 (1983);

A.V.Nesterenko and A.V.Radyushkin, Phys. Lett. B 115, 410 (1982).

[28] P.Colangelo, G.Nardulli, A.A.Ovchinnikov and N.Paver, Phys. Lett. B 269, 204 (1991).

[29] For a review see:

P.Colangelo, G.Nardulli and N.Paver, Proceedings of the ECFA Workshop on a European B-Meson Factory, R.Aleksan and A.Ali Eds., ECFA 93/151, pag.155.

C.A.Dominguez, preprint SISSA Ref 139/93/EP (hep-ph/9309260), to appear in the Proceedings of the Third Workshop on the Tau-Charm Factory, J.Kirkby and R.Kirkby Eds., Editions Frontieres, 1994.

[30] For a review see:

N.G.Deshpande, in: B decays, ed. S.Stone (World Scientific, 1992) p.430;

S.Bertolini, F.Borzumati and A.Masiero, in: B decays, ed. S.Stone (World Scientific, 1992) p.458;

A.Ali, C.Greub and T.Mannelm Proceedings of the ECFA Workshop on a European B-meson factory, R.Aleksan and A.Ali ed., ECFA 93/151, DESY 93-053.

[31] S.Bertolini, F.Borzumati and A.Masiero, Phys. Rev. Lett. 59, 180 (1987)

N.G.Despande, P.Lo, J.Trampetic, G.Eilam and P.Singer, Phys. Rev. Lett. 59, 183 (1987);

B.Grinstein, R.Springer and M.B.Wise, Nucl. Phys. B339, 269 (1990);

G.Cella, G.Curci, G.Ricciardi and A.Viceré, Phys. Lett. B248, 181 (1990);

M.Misiak, Phys. Lett. B 269, 161 (1991);

M.Ciuchini, E.Franco, G.Martinelli, L.Reina and L. Silvestrini, Phys. Lett. B316, 127 (1993) and hep-ph-9406239.

[32] N.Isgur and M.B.Wise, Phys. Rev. D 42, 2388 (1990).

[33] P.Colangelo, C.A.Dominguez, G.Nardulli and N.Paver, Phys. Lett. B 317, 183 (1993).

[34] A.Ali, V.M.Braun and H.Simma, preprint CERN-TH-7118-93,MPI-PH-93-97, DESY 93-193, hep-ph/9401277.

[35] R.Ammar et al. (CLEO Collab.), Phys. Rev. Lett. 71, 674 (1993).

[36] P.Santorelli, Z. fur Phys., C 61, 449 (1994).

[37] N.Isgur, D.Scora, B.Grinstein and M.B.Wise, Phys. Rev. D 39, 799 (1989). P.J.O'Donnell and H.K.K.Tung, Phys. Rev. D 44, 741 (1991). 


\section{TABLES}

TABLE I. The form factor $A_{0}(0)$ of various transitions in different models.

\begin{tabular}{llllllll}
\hline \hline Transition & {$[7]$} & {$[13$} & {$[14$} & {$[16]$} & {$[18$} & {$[19]$} & This paper \\
\hline$D \rightarrow K^{*}$ & 0.74 & 0.91 & 0.8 & $0.45 \pm 0.30$ & $0.77 \pm 0.29$ & 0.59 & $0.58 \pm 0.05$ \\
$D \rightarrow \rho$ & 0.68 & - & 0.85 & $0.57 \pm 0.40$ & - & 0.74 & $0.52 \pm 0.05$ \\
$B \rightarrow \rho$ & 0.28 & - & 0.14 & $0.79 \pm 0.80$ & $-0.57 \pm 0.65$ & 0.24 & $0.24 \pm 0.02$ \\
\hline \hline
\end{tabular}




\section{FIGURE CAPTIONS}

Fig. 1

The form factor $A_{0}^{D \rightarrow K^{*}}\left(q^{2}\right)$. The curves refer to different sets of parameters: $s_{0}=7 \mathrm{GeV}^{2}$ and $s_{0}^{\prime}=1.5 \mathrm{GeV}^{2}$ (continuous line), $s_{0}=7 \mathrm{GeV}^{2}$ and $s_{0}^{\prime}=1.7 \mathrm{GeV}^{2}$ (dashed line), $s_{0}=8 \mathrm{GeV}^{2}$ and $s_{0}^{\prime}=1.5 \mathrm{GeV}^{2}$ (dotted line), $s_{0}=8 \mathrm{GeV}^{2}$ and $s_{0}^{\prime}=1.7 \mathrm{GeV}^{2}$ (dasheddotted line). $M^{2}=3 \mathrm{GeV}^{2}, M^{\prime 2}=1.5 \mathrm{GeV}^{2}$.

Fig. 2

The form factor $A_{0}^{D \rightarrow \rho}\left(q^{2}\right)$. The curves refer to the sets of parameters: $s_{0}=7 \mathrm{GeV}^{2}$ and $s_{0}^{\prime}=1.3 \mathrm{GeV}^{2}$ (continuous line), $s_{0}=7 \mathrm{GeV}^{2}$ and $s_{0}^{\prime}=1.5 \mathrm{GeV}^{2}$ (dashed line), $s_{0}=8 \mathrm{GeV}^{2}$ and $s_{0}^{\prime}=1.3 \mathrm{GeV}^{2}$ (dotted line), $s_{0}=8 \mathrm{GeV}^{2}$ and $s_{0}^{\prime}=1.5 \mathrm{GeV}^{2}$ (dashed-dotted line). $M^{2}=3 \mathrm{GeV}^{2}, M^{2}=1.5 \mathrm{GeV}^{2}$.

Fig. 3

The form factor $A_{0}^{B \rightarrow \rho}\left(q^{2}\right)$. The curves refer to the sets of parameters: $s_{0}=33 \mathrm{GeV}^{2}$ and $s_{0}^{\prime}=1.3 \mathrm{GeV}^{2}$ (continuous line), $s_{0}=33 \mathrm{GeV}^{2}$ and $s_{0}^{\prime}=1.5 \mathrm{GeV}^{2}$ (dashed line), $s_{0}=36 \mathrm{GeV}^{2}$ and $s_{0}^{\prime}=1.3 \mathrm{GeV}^{2}$ (dotted line), $s_{0}=36 \mathrm{GeV}^{2}$ and $s_{0}^{\prime}=1.5 \mathrm{GeV}^{2}$ (dasheddotted line). The values of the Borel parameters are: $M^{2}=8 \mathrm{GeV}^{2}, M^{\prime 2}=2 \mathrm{GeV}^{2}$. 
This figure "fig1-1.png" is available in "png" format from: http://arxiv.org/ps/hep-ph/9409438v1 
This figure "fig1-2.png" is available in "png" format from: http://arxiv.org/ps/hep-ph/9409438v1 
This figure "fig1-3.png" is available in "png" format from: http://arxiv.org/ps/hep-ph/9409438v1 